\title{
Erdheim-Chester Disease : a Rare Disease Presenting with Multiple Jaw Lesions in a Child
}

\author{
Yasushi Morimoto ${ }^{1,2)}$, Phuu Pwint Han ${ }^{2)}$, Naoki Katase ${ }^{2)}$, Ryo Tamamura ${ }^{2)}$, Anak Iamaroon ${ }^{3)}$, \\ Lee Jeong-Keun ${ }^{4}$, and Hitoshi Nagatuska ${ }^{2)}$
}

1) Japan Institute for Advanced Dentistry, Osaka

${ }^{2)}$ Okayama University, Graduate School of Medicine, Dentistry and Pharmaceutical Sciences

${ }^{3)}$ Chang Mai University, Department of Odontology and Oral Pathology, Thailand

${ }^{4)}$ Ajou University, Department of Dentistry, Korea

\begin{abstract}
Clinical summary: A 13 years old female patient came to the Okayama University Hospital with a complaint of pain and swelling at the left side of the mandible. Radiological examination revealed a large welldefined radiolucent lesion extending from the mesial of tooth 35 to distal or 37 tooth(Fig.1A). Lingual plate perforation was also detected by CT scan (Fig.1B). With the provisional diagnosis of ameloblastoma, biopsy was done. The biopsy of the mandibular lesion was suspected of non-ossifying fibroma. Total curettage of the lesion was performed and post-operatively the healing was uneventful. During the follow-up period, 6 months after the operation, the patient complained of discomfort at the left maxillary region and epileptic attacks. Through examination of the patient by X-ray, CT scan and MRI revealed multiple bone lesions in the maxillae, tibias and fibulas bilaterally and a brain lesion. Curettage of the maxillary lesions was performed. She also underwent her brain surgery and the lesion removed was diagnosed as juvenile xanthogranuloma. The long bone lesions were only under surveillance and no intervening treatment was done. In the most recent followup, the patient was diagnosed to have an abdominal lesion as well.
\end{abstract}

\section{Microscopic findings}

The mandibular lesion contained spindle-shaped cells arranged in short fascicles forming whorl pattern, extensive areas of necrosis and xanthogranulomatous areas with hemorrhages and haemosiderin deposits. The maxillary lesions showed more prominent whorl pattern (Fig.2,A). Small mononuclear cell foci, Touton type giant cells and xanthomatous cells were also seen (Fig.2,B).

\section{Immunohistochemical findings}

Immunohistochemical study showed strong and diffuse positivity for CD68 (Fig.2,C) and vimentin but non-reactive to CK, desmin, NF, S-100 and CD1a (Fig.2,D).

\section{Electron microscopic findings}

Electron microscopic study revealed biphasic cells: histiocytic cells with irregular indented nuclei and many lipid vacuoles and lysosomes within their cytoplasms (Fig.3,A) and fibroblastic cells with interspersed collagen fiber between them. Birbeck's granules were not detected (Fig.3,B).

\section{References}

1. Chester W. Ü ber Lipoidgranulomatose Virchow. Arch Pathol Anat 1930
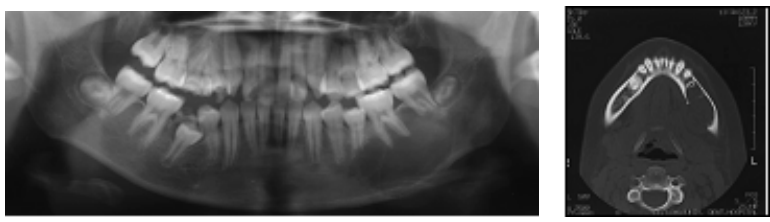

Fig.1 (A) Panoramic radiograph (B) CT scan at the first admission
2. Veyssier-Belot $\mathrm{C}$ et al. Erdheim-Chester disease clinical and radiologic characteristics of 59 cases. Medicine 1996

3. Jaffe R. The histiocytoses: review. Clin Lab Med 1999

4. Chetritt J et al. Chester-Erdheim disease: a neoplastic disorder. Hum Pahtol 1999

5. Al-Quran $\mathrm{S}$ et al. Erdheim-Chester disease: case report, PCR analysis of clonality and review of literature. Mod Pathol 2002

6. Nagatsuka $\mathrm{H}$ et al. Erdheim-Chester disease in a child presenting with multiple jaw lesions. J Oral Pathol Med 2005
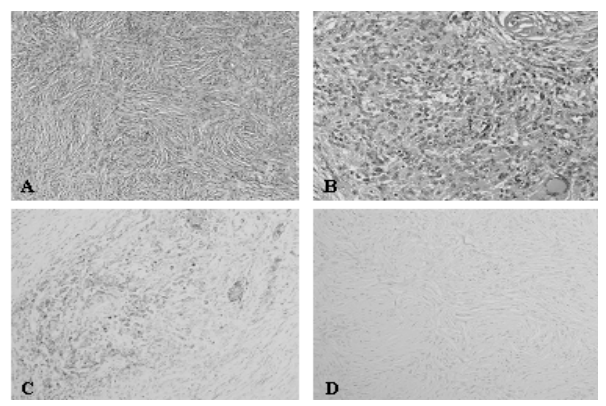

Fig.2 Maxillary lesion (A) Prominent whorl area (B) Xanthogranulomatous area with Touton type giant cells (C) CD68 (D) CD1a

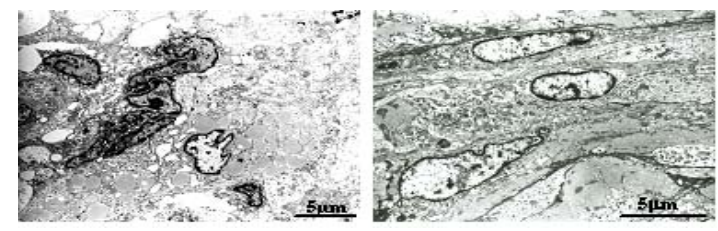

Fig.3 Electron microscopic findings (A) Histiocytic areas (B) Fibroblastic areas 